\title{
Content Analysis of the Meaning of Spiritual Care as Perceived by Nursing Students
}

\author{
Sun-Hwa Shin, R.N., Ph.D., Hyeon-Young Kim, R.N., Ph.D., Hee-Yeong Woo, R.N., Ph.D.*, \\ Myung-Nam Lee, R.N., Ph.D. ${ }^{\dagger}$ and Ye-Jean Kim, R.N., Ph.D.* \\ College of Nursing, Sahmyook University, "School of Nursing, Sahmyook Health University, Seoul, \\ ${ }^{\dagger}$ Department of Nursing, College of Health Science, Kangwon National University, Samcheok, Korea
}

Purpose: This study aimed to describe the meaning of spiritual care as perceived by nursing students. Methods: This study used a descriptive research design, and the participants were 126 fourth-year nursing students from three nursing colleges. Data were collected from $\mathrm{Au}^{-}$ gust to September 2019, and were analyzed using the content analysis method. Results: Four themes of spiritual care with 15 sub-themes were extracted from the content analysis: 1) "promoting spiritual well-being" (sub-themes: "providing religious help", "caring for the patient as a spiritual being", and "presupposing human dignity regardless of religion”); 2) "taking place in actual nursing practice" (representative sub-themes: "considering the perspective of the patient", "reducing suffering"); 3) "caring for the multifaceted needs of human beings" (representative sub-themes: "providing physical, mental, and spiritual care", "caring for both the mental and physical health of the patient"), and 4) "growing together" (sub-themes: "positively affecting patient well-being", "beginning with the nurse's selftranscendence"). Conclusion: These results suggest that nursing students consider spiritual care to be a highly positive and practical form of nursing care. However, because few students have been exposed to religion and spirituality, more systematic training should be provided.

Key Words: Spirituality, Nursing, Students, Perception
Received May 21, 2020

Revised June 22, 2020

Accepted July 21, 2020

Correspondence to

Ye-Jean Kim

ORCID:

https://orcid.org/0000-0001-5193-344X

E-mail:yejean73@shu.ac.kr

\section{INTRODUCTION}

\section{Background}

All humans are holistic beings with physical, mental, and spiritual dimensions, possessing spirituality, which drives the search for meaning and purpose of life and fundamentally integrates the various dynamics of human beings [1,2]. Spirituality constitutes the core element of a human being and represents the essence and purpose of being human [3,4], although it is a complex concept that may hold different meanings for different people [5]. Spirituality is also an integrated manifestation of individuals' education and experiences, reflecting their attitudes and behaviors in daily life [6], and is closely associated with health and nursing care [7].

Nursing is a holistic process of providing care for humans, in which each patient who receives nursing care should be seen as a being in the world with physical, mental, and spiritual capabilities. It should also provide spiritual care that harmoniously aligns with the mental, physical, and spiritual aspects of the patient, rather than simply focusing on physical treatment [8]. Spiritual care means providing care that meets a person's spiritual needs 
by sympathizing with his or her pain points [3,6,9]. Spiritual needs are among the deepest desires of humans, and fulfilling these needs allows us to stay hopeful at all stages of life and function with autonomy $[2,10]$. Spiritual care is also one of the most fundamental areas of nursing, as positive health outcomes are facilitated by meeting a patient's [5]. Spiritual nursing care can help patients foster positive attitudes toward treatment, as well as their life and death [11]. Fulfilling the spiritual needs of patients through spiritual nursing care not only helps to heal the patient, but also leads to positive results in terms of the spiritual well-being of their families [12]. Despite these advantages of providing spiritual care, which treats humans as holistic beings, nurses often feel a lack of confidence in addressing spiritual needs, as they are underestimated as simply being religious needs [12], and a generally passive attitude prevails when it comes to evaluating patients' spiritual needs; instead, there is a tendency to provide care mainly focused on the patient's physical needs $[13,14]$. Nursing practice that closely pays attention to the patient's spiritual needs is necessary for implementing holistic nursing care practices given that cancer patients-and others who require integrated nursing care-are more likely to struggle with maintaining spiritual well-being and to have unfulfilled spiritual needs [15].

Healthcare workers are in a critical position to meet patients' spiritual needs when treating and providing care $[9,13,16]$. Nurses, in particular, have the responsibility to heal patients, to provide an intuitive sense, to search for spiritual perspectives, to carry out patient-centered and meaning-centered treatment, and to create an environment that fosters spiritual growth by serving as healing agents [12]. As such, spiritual care means identifying the spiritual resources of the patients, who are holistic beings, and performing meaning-centered nursing through a person-centered approach [4]. The healing environment created by the spiritual care provided by nurses can also improve patients' health and well-being $[9,11]$. While many healthcare workers wish to provide spiritual care for patients, nurses and doctors agree that insufficient training on the subject is a barrier to providing spiritual care $[13,17]$. Research has also shown that one of the reasons that nurses fail to provide spiritual care is a lack of information and clear definitions of the concept of spirituality and spiritual care [12,13]. Spiritual nursing care will become an increasingly essential role of nurses, and their responsibility to perform holistic nursing will become more important [18]. It is vitally necessary to perform spiritual care that utilizes resources based on spirituality $[3,9,18]$. To do so, nursing college students should be taught about the concept of spiritual care so that they can develop their competency to provide spiritual care through systematic learning and training to become nurses who actively utilize spiritual resources [19,20].

A study of 500 nursing college students demonstrated that they perceived spirituality as an essential part of leading a happy human life $[19,21]$. Another study showed that students who completed courses on spirituality and/or spiritual nursing had higher levels of interest in nursing and a stronger will to become nurses [6]. Senior nursing students reported the power of practicing spiritual nursing by testifying how they were able to become more spiritually mature through the emotionally touching and rewarding process of clinical spiritual nursing practice, overcoming their initial emotional burden of anxiety and high expectations [20].

Although spiritual care has received more attention as educational programs have increasingly dealt with spirituality and spiritual nursing competencies, little research has been done to understand nurses' and nursing students' actual awareness of spiritual care. Learning and becoming aware of spiritual care through theoretical studies and clinical practice will serve as the foundation for nursing students to provide spiritual care as actual nurses. Against this backdrop, this study sought to verify the meaning of spiritual care experienced by nursing students through their clinical practice course. The purpose of this study was to understand how senior nursing students perceived spiritual care and to identify their perceptions of the meaning of spiritual care. Hopefully, the findings of this study will serve as the foundation for developing a curriculum focused on spirituality and strengthened spirituality training.

\section{Conceptual foundation}

The conceptional foundation of this study is based on Frankl's [1] concept of logotherapy, represented by the "will to meaning." The premise of this theory is that humans are the subjects of care, and are spiritual beings who have the responsibility to realize the unique meaning of their lives and to achieve transcendence, and the primary motivational force of 
an individual is to find meaning in life [1]. Nursing students need to prepare for providing spiritual care and will be able to administer spiritual care when they find the motivation to find the meaning of spiritual care. Based on this foundation, the researchers created a set of questions consisting of two specific domains - the meaning of spiritual care and the practice of spiritual care-informed by the framework of logotherapy to research the experiences of nursing students with spiritual care (Figure 1).

\section{METHODS}

\section{Study design}

This study used qualitative content analysis to categorize the content of nursing students' perceptions regarding the meaning of spiritual care.

\section{Study participants}

The participants of this study were senior nursing students who were able to express their opinions on the subject based on their clinical practice course experiences. Participants were selected using convenience sampling among undergraduate students of a nursing college and a nursing community college in Seoul, and undergraduate students of a nursing college in Gangwon Province. Participants were selected with the goal of representing different opinions across regions, schools, and religions to obtain insights into diverse opinions on spiritual care. A total of $126(46.7 \%)$ senior students participated in this study. The process of sampling participants is shown in Figure 2.

\section{Data collection and ethical considerations}

Content analysis can be performed using methods such as open-ended surveys, individual and focus group interviews, observations, or analyses of the literature, including books and articles [22]. In this study, we designed an open-ended survey

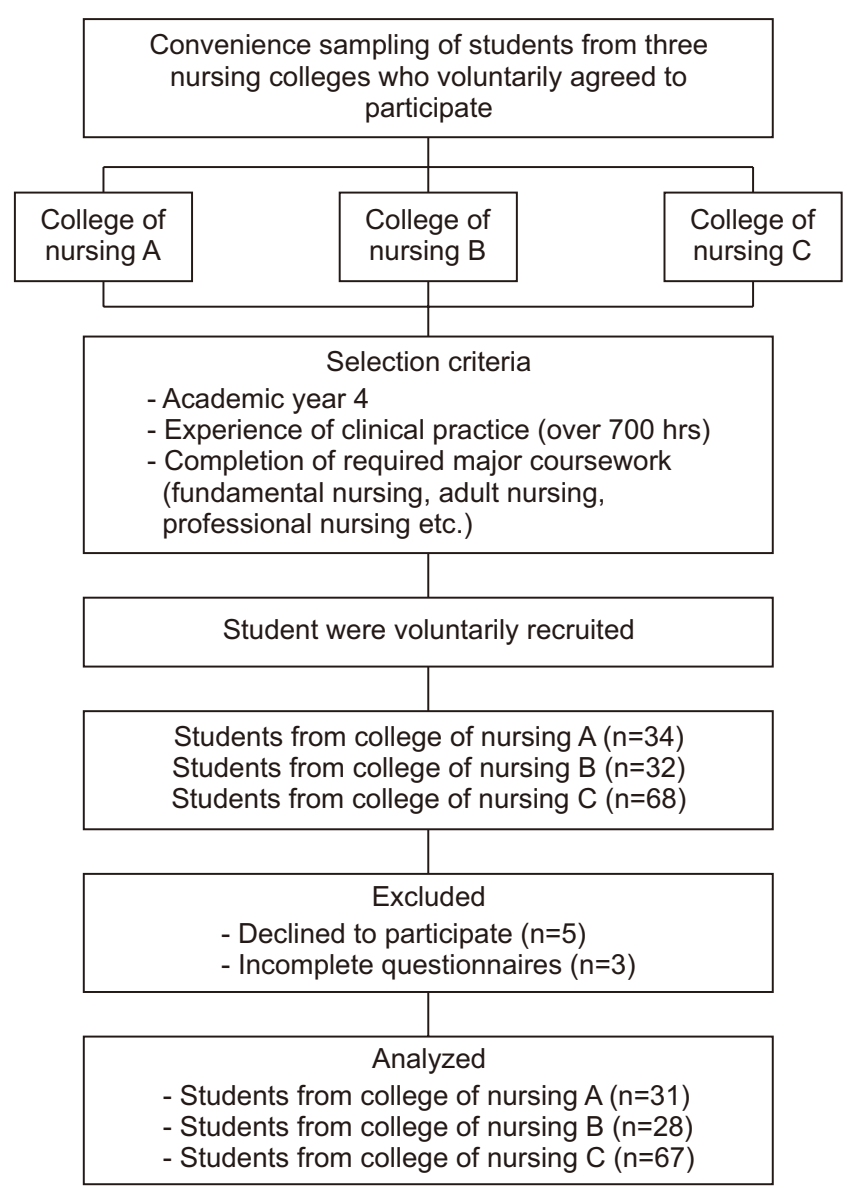

Figure 2. The sampling schema of the present study.

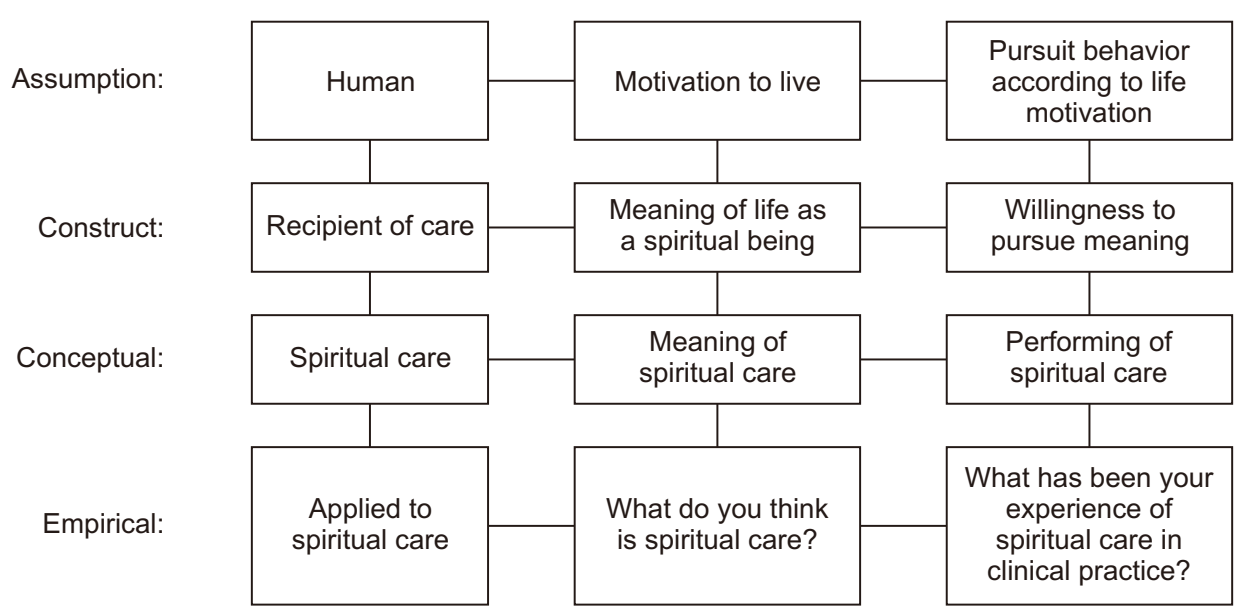

Figure 1. Conceptual framework for constructing the research questions. 
including two questions: "In your opinion, what is spiritual care?" and "What has been your experience with spiritual care in the clinical practice setting?" These questions were created based on the concepts of logotherapy and motivation theory (focusing on the meaning and the performance of spiritual care) to identify the content of nursing students' perceptions of the meaning of spiritual care.

Data collection was carried out between the end of $\mathrm{Au}^{-}$ gust and mid-September in 2019. The study was approved by the Institutional Review Board of $S$ University to protect the participants (Approval number: 2-7001793-AB-N012019078HR). Data were collected from participants who volunteered to participate, and participants described their opinions freely in the anonymous open-ended survey questions.

One of the researchers explained the purpose of the study in the classroom prior to distributing the open-ended questionnaire, including the research purpose and procedures, the right to discontinue participation in the research, and the potential benefits and disadvantages of participating in the study.

We ensured that the subjects spontaneously participated in the study based on their understanding of the purpose and had a research assistant help with the study to ensure maximum autonomy in study participation. The research assistant distributed the survey and asked the participants to complete it after taking time to collect their thoughts on their perceptions of the meaning of spiritual care. On average, participants took 30 to 45 minutes to complete the survey, and those who needed additional time were told to submit their responses to the assistant later.

\section{Data analysis}

The content analysis method was used to examine the nursing students' perceptions of the meaning of spiritual care.

The collected survey data were analyzed using the following method: First, each researcher extracted sentences considered to be relevant to the meaning of spiritual care from the participants' responses, and then the compared the 389 selected sentences head-to-head and identified the final sentences after repeated reviews of the original text submitted by the participants. Second, the researchers selected the final 352 sentences after reviewing previously selected sentences that were thought to convey the meaning of spiritual care, through two meetings. Third, the 352 sentences were categorized into sub-themes with similar content. Twenty-four sub-themes were initially identified, but were reduced to 15 after three rounds of discussion. The fourth and final step was conceptualizing the subthemes to form more comprehensive themes. Through multiple rounds of discussion, six initially selected themes were reduced to four final umbrella themes. Each theme was categorized based on the statements submitted by participants. The number of core sentences by each participant ranged from 1 to 6 , and the frequency and percentage of significant findings within each theme were calculated using quantitative content analysis. The data were evaluated by four nursing professors who had 8 to 20 years of experience teaching at nursing colleges, as well as experience in qualitative research and content analysis. The actual coding of the data was performed after many calibration sessions until the agreement among the researchers met the $90 \%$ threshold needed to achieve an adequate confidence level. Any mismatches or excluded sentences in the outcomes were discussed among the researchers during the coding process, resulting in the decision to delete the sentence or to propose a new category. The level of agreement among researchers when categorizing the content was calculated [23], and Holsti's method of intercoder reliability showed a high level of agreement (94\%). The credibility of the outcomes of this study was carefully ensured by having researchers maintain a neutral stance when analyzing the data, having an nursing professor with experience in content analysis in the field confirm the validity of the final themes and sub-themes, and by confirming the content with participants.

\section{RESULTS}

\section{General characteristics of the participants}

Twenty-nine participants were men (23.0\%) and 97 were women (77.0\%). Their age distribution was as follows: 21 years $(n=9,7.1 \%), 22$ years $(n=43,34.1 \%), 23$ years $(n=32$, $25.4 \%), 24$ years $(n=15,11.9 \%), 25$ years $(n=16,12.7 \%), 26$ years $(n=5,4.0 \%)$, and $27 \sim 30$ years $(n=6,4.8 \%)$. Fifty-seven participants were Protestants (45.2\%), 52 had no religious beliefs (41.4\%), seven were Catholics (5.6\%), three were Bud- 
dhists (2.4\%), and seven gave no response (5.6\%) (Table 1).

\section{Content analysis of spiritual care}

The content analysis of nursing students' perceived meaning of spiritual care were summarized into 352 significant findings, 15 sub-themes, and four themes (Table 2). The details of the extracted themes and sub-themes are outlined in this section.

\section{1) Theme 1. Promoting spiritual well-being}

The theme of "promoting spiritual well-being" had the subthemes of "providing religious help", "caring for the patient as a spiritual being", and "presupposing human dignity regardless of religion."

"Providing religious help" was the most frequently appearing sub-theme (52 times, 14.8\%), of which the significant findings included "respecting the patient's religion", "providing care based on his or her own religion", "connecting with religious leaders", "helping to build a relationship with a religious authority", and "praying as religious meditation." This means acknowledging the beliefs of the patient and the nurse and focusing on the religious behavior of helping them build a relationship with an absolute being. "Caring for the patient as a spiritual being" was found in $36(10.2 \%)$ sentences, with significant findings including "responding to the patient's spirituality beyond religion", "helping the patient to lead a spiritual existence", "identifying and meeting spiritual needs", and "helping to maintain spiritual well-being." This sub-theme

Table 1. General Characteristics ( $N=126)$.

\begin{tabular}{clrr}
\hline Characteristics & Categories & $\mathrm{n}$ & $\%$ \\
\hline \multirow{4}{*}{ Sex } & Male & 29 & 23.0 \\
& Female & 97 & 77.0 \\
& 21 & 9 & 7.1 \\
& 22 & 43 & 34.1 \\
& 23 & 32 & 25.4 \\
& 24 & 15 & 11.9 \\
Religion & 16 & 12.7 \\
& 25 & 5 & 4.0 \\
& 26 & 6 & 4.8 \\
& None & 52 & 41.3 \\
& Protestantism & 57 & 45.2 \\
& Catholicism & 7 & 5.6 \\
& Buddhism & 3 & 2.4 \\
& No answer & 7 & 5.6 \\
\hline
\end{tabular}

dealt with helping the patient develop his or her spiritual resources as a spiritual being by identifying their spiritual needs beyond religion. "Presupposing human dignity regardless of religion" appeared in 28 sentences (8.0\%), of which the significant findings included "preserving human dignity regardless of religion", "providing care based on the value of the human being", and "respecting the patient's choices and human rights." This sub-theme involved helping patients find their meaning of life and happiness based on human dignity, not limited to religion.

\section{2) Theme 2. Taking place in actual nursing practice}

A second theme relating to the meaning of spiritual care as perceived by nursing students was "taking place in actual nursing practice", which included "considering the perspective of the patient", "reducing suffering", "providing the care required for the dying patient", "communicating fully", "meeting the patient's primary needs", and "providing care in anticipation of problems/changes."

Among these sub-themes, "considering the perspective of the patient" appeared in 37 findings (10.5\%), including "focusing on the patient's mental well-being and providing comfort", "approaching the patient sincerely and with affection", and "considering and supporting the patient." This sub-theme involved sympathizing with the patient and providing proactive help to comfort him or her. "Reducing suffering" included 28 (8.0\%) findings, such as "understanding the patient's suffering and responding", "reducing the patient's anxiety", and "relieving stress and tension." This perception viewed the meaning of spiritual nursing as being about relieving the suffering experienced by patients, including anxiety, pain, nervousness, and stress, to help them maintain their dignity. "Providing the care required for the dying patient" encompassed 20 findings (5.7\%), including "providing essential care for the dying patient" and "providing peaceful end-of-life care." From this standpoint, spiritual care is a type of nursing care that is essential for patients nearing death. Thirteen (3.7\%) findings fell under the sub-theme of "communicating fully", including "performing therapeutic communication techniques", "using natural communication to help patients restore relationships", and "promoting the patient's relationships with others." This sub-theme referred to communicating with the patient through 


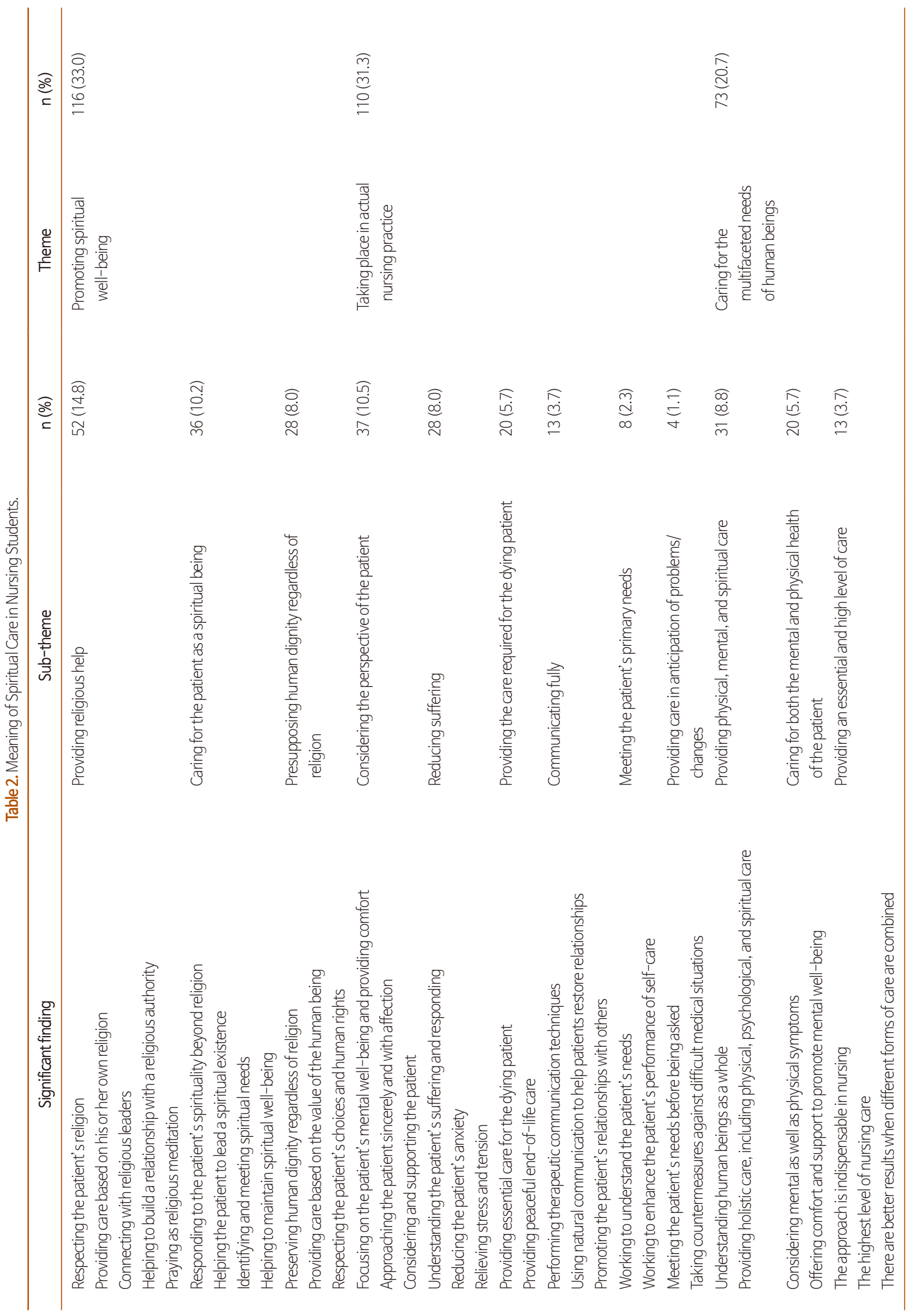


extensive conversations to seek to restore their personal relationship. "Meeting the patient's primary needs" encompassed eight (2.3\%) findings including "working to understand the patient's needs", and "working to enhance the patient's performance of self-care." This sub-theme included actions taken to meet the patient's needs to promote recovery of the patient. "Providing care in anticipation of problems/changes" was found in 4 (1.1\%) responses, which included "meeting the patient's needs before being asked", and "taking countermeasures against difficult medical situations." This referred to predicting potential problems and providing proactive care.

\section{3) Theme 3. Caring for the multifaceted needs of human beings}

The sub-themes of "providing physical, mental, and spiritual care", "caring for both the mental and physical health of the patient", "providing an essential and high level of care", and "including all involved persons" were all summarized under the umbrella theme of "caring for the multifaceted needs of human beings."

Among these sub-themes, "providing physical, mental, and spiritual care" appeared in 31 (8.8\%) findings, including "understanding human beings as a whole" and "providing holistic care, including physical, psychological, and spiritual care." This referred to the provision holistic care encompassing the physical, mental, and spiritual aspects of patients. "Caring for both the mental and physical health of the patient" was found in $20(5.7 \%)$ findings, including "considering mental as well as physical symptoms", and "offering comfort and support to promote mental well-being." This sub-theme described a focus on patients' physical and mental health. Thirteen (3.7\%) findings were categorized into "providing an essential and high level of care", including "the approach is indispensable in nursing", "the highest level of nursing care", and "there are better results when different forms of care are combined." This means that spiritual care is a high-level area of nursing with broad impacts. "Including all involved persons" encompassed nine significant findings (2.6\%), including "the approach is necessary for all patients, not just those who practice religion", "the patient and the family heal together", "the approach includes not only nurses, but also patients, caregivers, and cleaning staff." In other words, spiritual care was perceived as 
a type of care provided not only provided to the patient, but also to everyone who can influence the patient.

\section{4) Theme 4. Growing together}

The significant findings "positively affecting patient wellbeing" and "beginning with the nurse's self-transcendence" were integrated under the sub-theme "growing together."

The sub-theme of "positively affecting patient well-being" was found in 45 (12.8\%) findings, including "enabling the nurse to find the meaning and purpose of life", "giving hope and resulting in increased willingness to heal", and "Involving healing from the depths of the heart." This referred to nurturing the patient's courage and the power of endurance by changing patients' perspective of life. The sub-theme "beginning with the nurse's self-transcendence" covered $2.3 \%(n=8)$ of the findings including "beginning with the nurse's self-care", "the nurse should take a spiritual look at himself/herself", and "the nurse needs to be willing to give of himself/herself." This implies that spiritual care for patients begins by maintaining the nurse's spiritual well-being.

\section{DISCUSSION}

This study aimed to identify the content of nursing students' perceptions of the meaning of spiritual care with the goal of providing a foundation for seeking ways to enhance spiritual care competencies in nursing education. Spiritual needs are a core desire of human beings and spiritual care is valuable given that meeting one's spiritual needs has positive effects in all stages of life [1,3-5]. This study found that the perceptions of spiritual care among nursing college students could be categorized into the following themes: "promoting spiritual well-being", "taking place in actual nursing practice", "caring for the multifaceted needs of human beings", and "growing together."

First, spiritual care was found to promote spiritual wellbeing. Most of the nursing students associated spiritual care with religion and found that religious care was a way of promoting spiritual well-being. The meaning and concept of religion were strongly associated with spiritual nursing education, in accordance with the findings of previous studies suggesting that spiritual nursing interventions focused on providing religious care such as biblical texts, hymns, prayers, and connect- ing with religious leaders [24]. However, this finding should be carefully interpreted, keeping in mind that $58.6 \%$ of the participants were religious. Participants in this study also stated that spiritual care is about caring patients as spiritual beings, beyond religion. This supports the findings of previous studies suggesting that spirituality in nursing should be seen as a more comprehensive concept of understanding the meaning of life transcending the material world, rather than simply as religious beliefs $[4,24]$. Nursing studies should take a more comprehensive approach to spirituality in terms of spiritual care, while of course including the religious context [25]. The study also found that the participants perceived spiritual care as respecting human dignity itself, not simply confined to religion. This is in line with a previous study suggesting that spiritual care provided to patients who are experiencing life-threatening or life-limiting disease seeks to provide holistic care to maintain human dignity and a high quality of life [5] and that medical workers treating patients in the final stage of their life should carefully attend to the spiritual well-being of the patients [13]. Nursing research in South Korea has largely focused on integrated and multifaceted interventions to understand the effects of spiritual care on patients' spiritual well-being, as well as the effects of logotherapy without a religious background, reminiscence therapy, well-rounded health interventions, education to prepare for death, empathy, and self-understanding [24]. Although some Christian nursing schools offer nursing clinical practice courses that include spiritual care from a religious perspective [20], other concepts of spirituality, spiritual being, and spiritual needs in terms of human dignity include the meaning and purpose of life, hope, and satisfaction. Further research should be carried out to promote spiritual nursing that cares for patients as spiritual beings, beyond religion, in clinical and nursing education settings.

Second, spiritual care is not an abstract concept; instead, it refers to specific actions performed as part of nursing practice in clinical settings. The sub-themes, in the order of the most frequent percentage of appearance, were "considering the perspective of the patient" ( $n=37)$, "reducing suffering" $(n=28)$, "providing the care required for the dying patient" $(n=20)$, and "communicating fully" $(n=13)$. This result is in accordance with previous studies suggesting that spiritual care is about listening and sympathizing with what patients have to say and 
building positive relationships [26]. It also aligns with another study reporting that nurses inevitably perform spiritual care when providing care for patients faced with the threat of death and extreme spiritual suffering [15]. The findings of this study also support some previous studies that defined the characteristics of spiritual care as utilizing one's self in the process of treatment (active listening, treatment relationship, and unconditional acceptance), intuitive senses (sensing spiritual conversations with patients), searching for a spiritual perspective (evaluating spirituality, evaluating the source of power and hope, and evaluating spiritual needs), patient-centered and meaning-centered interventions (building meaningful relationship, hope, and religious interventions), and creating a spiritual environment (respecting spiritual beliefs, ethical treatment, and respecting cultural and spiritual values) [12]. Spiritual care interventions include more direct "doing" such as praying, listening, requesting for religious help from a pastor, and providing reading materials, as opposed to "being", which is about maintaining the relationship of nurse and patient while utilizing resources such as individual capabilities or personality [27]. The findings of this study on the specific content of spiritual care through nursing students' statements include smooth communication, friendly words, abundant conversation, consoling words, and warm eye-contact. These are part of the practice of communication in treatment by nurses who spend extensive time in clinical settings, and reflect realistic and hands-on nursing practices that can help fulfill patients' spiritual needs. Nursing students also stated that spiritual care is essential for patients in the face of death. These findings confirm that helping patients on their deathbeds to die peacefully is an essential part of nursing in the context of spiritual care. Previous studies found that patients who received sufficient support for their spiritual needs from healthcare providers were 3.53 times more likely to accept hospice care than other patients, and spiritual care was found to be a crucial factor in enhancing the quality of life of patients facing death [28], which has the same implications as the findings of this study. Other findings included meeting patients' major needs $(n=8)$, and nursing them by predicting issues and changes $(n=4)$, confirming that spiritual care involves providing nursing care based on identifying the needs and potential issues of patients. Therefore, more detailed guidance regarding the $\mathrm{ac}^{-}$ tions of spiritual care performed in clinical settings and clinical guidance for spiritual nursing should be developed.

Third, the participants described spiritual care as caring for the multifaceted needs of human beings. This included providing physical, mental, and spiritual care $(n=31)$, as well as caring for both the mental and physical health of the patient $(n=20)$. Most of the students perceived spiritual care as an integrated approach that is not limited to a single aspect, while some students perceived spiritual care as limited to the mental/ physical aspect of care, without considering the spiritual element. The participants in this study mostly perceived spiritual care as holistic nursing that encompasses the spiritual aspect and stated that a comprehensive spiritual care is a high-level form of essential care that should be provided to all patients. This mirrors the conclusions of existing studies that nurses should be aware of all physical, mental, and spiritual elements to meet patients' needs for holistic nursing and should implement a comprehensive approach for balanced interventions $[9,16,18]$. This also supports the claim of a previous study that spiritual care is the only subjective and dynamic method that integrates all the aspects above [12]. The main novel finding of this study is that subjects of holistic care should include both patients and those who share space with patients, such as family members, nurses, caregivers, and janitors. This implies the need for holistic nursing that addresses the spiritual well-being of family and professional caregivers who spend time with patients. Future studies should probe the scope and extension of the concept of holistic nursing given that holistic nursing is currently focused on physical, mental, and spiritual care for patients only $[16,18]$. Moreover, standardized spiritual nursing education is desperately needed to improve the understanding of the spiritual aspects of subjects, as we found that some students perceived spiritual care as limited to physical and mental aspects. It is important for nursing students to improve their spiritual awareness so that they can meet $\mathrm{pa}^{-}$ tients' spiritual needs, and proper training should be provided to improve their perspectives on spirituality [21]. Developing a standardized curriculum and educational guidance for spiritual nursing for nursing students to help them learn about spirituality and spiritual care would help build a consistent system across theoretical and clinical settings.

Fourth, spiritual care not only helps the patient, but also 
helps the nurse grow together with the patient. Patients who received spiritual care saw positive changes, finding a specific meaning and purpose of life, as well as hope. Just as spirituality and self-transcendence give humans the power to overcome frustrating circumstances, improve confidence in life, and empower self-healing [26], it is reasonable to assume that at least some patients who receive spiritual care experience spiritual transcendence. As such, spiritual care is a crucial aspect of treating patients, and good spiritual care may yield positive results both for patients and for medical service providers $[12,18]$. This study found that nurses should have the experience of self-transcendence by spiritually caring for themselves, in addition to bringing positive changes for patients. Existing studies of nursing students showed that the clinical practice of spiritual nursing gave them the chance to become more mature by realizing weak spots of their spiritual condition and reflecting on themselves [20]. Additionally, the spiritual care ability of nurses who received spiritual intelligence training improved positively [17]. Nurses can provide spiritual care by perceiving their own spirituality, as well as that of the patient [14]. Nurses can experience self-transcendence by practicing spiritual care, as they play an important role in supporting patients who experience spiritual suffering or express spiritual needs [27]. This experience of self-transcendence not only helps them grow, but also corresponds to the beginning of spiritual care, through which nurses assist in the recovery of patients' health by practicing true interest and love towards the patients. It was confirmed that the self-transcendence of nurses, as $\mathrm{de}^{-}$ scribed in this study, can serve as a driving force to overcome the difficulties in their lives by helping them to understand their spiritual needs and to discover the purpose and meaning of life. As we can see, nurses should possess a clear perspective on spiritual care to practice spiritual nursing and maintain a high level of spiritual well-being for themselves [4,11]. In light of these findings, experience-based content should be included in spiritual nursing education to help students improve their spiritual sensitivity through spiritual training and experience self-transcendence. Spiritual nursing training will help students grow into nurses who improve their spiritual nursing competencies to practice patient-centered spiritual care based on their own experiences of self-transcendence.

The findings of this study should be carefully interpreted, as the survey was only conducted among students attending three nursing colleges. The key suggestions based on the findings of this study are as follows: First, we suggest that future studies should research the diverse range of variables that can affect the spiritual nursing competencies of nursing students. Second, we suggest the development of a standardized education program that promotes spirituality and spiritual nursing competencies. Third, we suggest that a similar content analysis should be conducted among nurses with experience in clinical settings for comparative purposes. Fourth, we suggest that a study should be conducted to develop a measurement tool to assess the status of spiritual health of nurses and nursing students.

\section{CONFLICT OF INTEREST}

No potential conflict of interest relevant to this article was reported.

\section{ORCID}

Sun-Hwa Shin, https://orcid.org/0000-0003-4052-9542

Hyeon-Young Kim, https://orcid.org/0000-0003-22306732

Hee-Yeong Woo, https://orcid.org/0000-0001-5588-2547

Myung-Nam Lee, https://orcid.org/0000-0002-7272-752X

Ye-Jean Kim, https://orcid.org/0000-0001-5193-344X

\section{AUTHOR'S CONTRIBUTIONS}

Study design: SHS, HYK, HYW, YJK. Data collection: SHS, YJK, MNL. Data analysis: SHS, HYK, HYW, YJK. Manuscript writing and revisions: SHS, HYK, HYW, MNL, YJK.

\section{SUPPLEMENTARY MATERIALS}

Supplementary materials can be found via https://doi. org/10.14475/kjhpc.2020.23.3.151. 


\section{REFERENCES}

1. FrankI VE. Man's search for ultimate meaning. New York: Perseus Pub.;c2000.

2. Taylor EJ. Prevalence and associated factors of spiritual needs among patients with cancer and family caregivers. Oncol Nurs Forum 2006;33:729-35.

3. Puchalski CM, Vitillo R, Hull SK, Reller N. Improving the spiritual dimension of whole person care: Reaching national and international consensus. J Palliat Med 2014;17:642-56.

4. Monareng LV. Spiritual nursing care: A concept analysis. Curationis 2012;35:28.

5. Timmins F, Caldeira S. Understanding spirituality and spiritual care in nursing. Nur Stand 2017;31:50-7.

6. Wu LF, Liao YC, Yeh DC. Nursing student perceptions of spirituality and spiritual care. Nurs Res 2012;20:219-27.

7. Meehan TC, Spirituality and spiritual care from a careful nursing perspective. J Nur Manag 2012;20:990-1001.

8. Watson J. Nursing : human science and human care. New York:National League for Nursing;1988. p.55-60.

9. Wright S, Neuberger J. Why spirituality is essential for nurses. Nurs Stand 2012;26:19-21.

10. Kang KA, Choi YS, Kim YJ. Reliability and validity of an instrument assessing spiritual needs of families of terminal cancer patients. Korean J Hosp Palliat Care 2018;21:144-51.

11. Seo EY, Kwon SH, Kim YK, Han AL. Experience of cancer patients receiving spiritual nursing care in one Christian general hospital. Asian Oncol Nurs 2019;19:179-91.

12. Ramezani M, Ahmadi F, Mohammadi E, Kazemnejad A. Spiritual care in nursing: A concept analysis. Int Nurs Rev 2014;61:211-19.

13. Balboni MJ, Sullivan A, Enzinger AC, Epstein-Peterson ZD, Tseng YD, Mitchell C, et al. Nurse and physician barriers to spiritual care provision at the end of life. J Pain Symptom Manage 2014;48:400-10.

14. Ellis HK, Narayanasamy A. An investigation into the role of spirituality in nursing. Br J Nurs 2009;18:886-90.

15. Astrow AB, Wexler A, Texeira K, He MK, Sulmasy DP. Is failure to meet spiritual needs associated with cancer patients' perceptions of quality of care and their satisfaction with care? J Clin Oncol 2007;25:5753-7.

16. Noh HK. Healthcare provider's spiritual care for the holistic healing. Personalism Bioethics 2015;5:87-123.

17. Riahi S, Goudarzi F, Hasanvand S, Abdollahzadeh H, Ebrahimzadeh F, Dadvari Z. Assessing the effect of spiritual intelligence training on spiritual care competency in critical care nurses. J Med and Life 2018;11:346-54.

18. Carpenter K, Girvin L, Kitner W, Ruth-Sahd LA. Spirituality: a dimension of holistic critical care nursing. Dimens Crit Care Nurs 2008;27:1620.

19. Daghan S. Nursing students' perceptions of spirituality and spiritual care; An example of Turkey. J Relig Health 2018;57:420-30.

20. Kwon SH, Tae YS. Christian nursing students' experience of spiritual nursing practice. J Qual Res 2013;14:92-104.

21. Tiew LH, Creedy DK, Chan MF. Student nurses' perspectives of spirituality and spiritual care. Nurse Educ Today 2013;33:547-79.

22. Son HM. Understanding and application of qualitative content analysis. J Korean Assoc Qual Res 2017;2:56-63.

23. Krippendorff K. Content analysis : an introduction to its methodology. 2nd ed. Beverly Hills, Calif.:London:Sage;c2004.

24. Yoo SY. Analysis of research trends about spiritual care in Korea. Korean J Adult Nur 2013;25:332-43.

25. Reinert KG, Koenig HG. Re-examining definitions of spirituality in nursing research. J Adv Nurs 2013;69:2622-34.

26. Kim KJ, Yong JS. Spirituality, death anxiety and burnout levels among nurses working in a cancer hospital. Korean J Hosp Palliat Care 2013;16:264-73.

27. Ilvia Caldeira S, Timmins F. Implementing spiritual care interventions. Nurs Stand 2017;31:54-60.

28. Balboni TA, Paulk ME, Balboni MJ, Phelps AC, Loggers ET, Wright AA, et al. Provision of spiritual care to patients with advanced cancer: associations with medical care and quality of life near death. J Clin Oncol 2010;28:445-52. 